\title{
On characteristic integrals of Toda field theories
}

Zhaohu Nie

To cite this article: Zhaohu Nie (2014) On characteristic integrals of Toda field theories, Journal of Nonlinear Mathematical Physics 21:1, 120-131, DOI:

https://doi.org/10.1080/14029251.2014.894724

To link to this article: https://doi.org/10.1080/14029251.2014.894724

Published online: 04 January 2021 


\title{
On characteristic integrals of Toda field theories
}

\author{
Zhaohu Nie \\ Department of Mathematics and Statistics, Utah State University, \\ Logan, UT 84322-3900, USA \\ zhaohu.nie@usu.edu
}

Received 14 August 2013

Accepted 4 December 2013

\begin{abstract}
Characteristic integrals of Toda field theories associated to general simple Lie algebras are constructed using systematic techniques, and complete mathematical proofs are provided. Plenty of examples illustrating the results are presented in explicit forms.
\end{abstract}

\section{Introduction}

First consider the famous Liouville equation, for which we take the following version:

$$
u_{x y}=-e^{2 u},
$$

where $x$ and $y$ are the independent variables, and $u=u(x, y)$ is an unknown function. Let

$$
I=u_{x x}-u_{x}^{2}
$$

Then it is easily checked that $I_{y}=\frac{\partial}{\partial y} I=0$ for a solution $u(x, y)$ to (1.1). $I$ is thus called a characteristic integral of (1.1).

Toda field theories are generalizations of the Liouville equation (1.1). Let $\mathfrak{g}$ be a simple Lie algebra of rank $n$ with Cartan matrix $A=\left(a_{i j}\right)_{i, j=1}^{n}$. For $1 \leq i \leq n$, let $u^{i}=u^{i}(x, y)$ be $n$ unknown functions of the independent variables $x$ and $y$. The Toda field theory associated to $\mathfrak{g}$ is the system of nonlinear PDEs:

$$
u_{x y}^{i}=-e^{\rho_{i}}:=-\exp \left(\sum_{j=1}^{n} a_{i j} u^{j}\right), \quad 1 \leq i \leq n .
$$

The Liouville equation (1.1) is the Toda field theory associated to $A_{1}=\mathfrak{s l}_{2}$.

Toda field theories are fundamental integrable systems with rich properties and important applications in mathematics and physics. They have been extensively studied, and we refer to the two books $[2,16]$ for surveys on them. In this paper, we are concerned with the explicit forms of their characteristic integrals.

Definition 1.1. A differential polynomial in the $u^{i}(x, y)$ for $1 \leq i \leq n$ is a polynomial in the $u^{i}$ and their partial derivatives with respect to $x$ of various orders $u_{x}^{i}, u_{x x}^{i}, \cdots$.

A characteristic integral of the Toda field theory (1.3) is a differential polynomial $I$ in the $u^{i}(x, y)$ for $1 \leq i \leq n$ such that $I_{y}=0$ for the solutions $u^{i}(x, y)$ to (1.3). 
By the symmetry between $x$ and $y$ in the Toda field theory (1.3), there are differential polynomials $\tilde{I}$ in the $u^{i}$ and their $y$-partial derivatives such that $\tilde{I}_{x}=0$ but they clearly follow the same patterns as those in the above definition.

Since a differential polynomial in characteristic integrals is another such integral, the characteristic integrals form a differential algebra. Shabat and Yamilov [19] showed that the characteristic integrals of the Toda field theory (1.3) form a polynomial differential algebra generated by $n$ primitive characteristic integrals, and that the system (1.3) has such a complete set of characteristic integrals if and only if the matrix $A=\left(a_{i j}\right)$ is equivalent to the Cartan matrix of a simple Lie algebra.

For a differential mononomial in the $u^{i}$, we call by its degree the sum of the orders of differentiation multiplied by the algebraic degrees of the corresponding factors. Therefore the $I$ in (1.2) has a homogeneous degree 2 . The primitive characteristic integrals can be chosen to be homogeneous and their degrees are equal to the degrees of the Lie algebra $\mathfrak{g}$. For a proof of this structure theorem, see [18] or [8, Theorem 2.4.10 and Proposition 2.4.7]. Recall that the algebra of adjoint-invariant functions on $\mathfrak{g}$ is a polynomial algebra on $n$ homogenous generators, whose degrees we call the degrees of $\mathfrak{g}[12]$. This paper is concerned with constructing these primitive characteristic integrals.

Many works $[3,5,7,16]$ have been devoted to the characteristic integrals, very often under different names such as local conservation laws, chiral currents, intermediate integrals or $W$-algebras and from different viewpoints. In particular, the works of Guryeva and Zhiber $[10,11]$ have shown that the Toda field theories (1.3) for classical and exceptional Lie algebras are of Liouville type, and have obtained explicit formulas for their generalized Laplace invariants, which can be used to construct the characteristic integrals and higher symmetries.

However to this author, the construction results about characteristic integrals for the Toda field theories (1.3) are not explicit enough, in terms of both the formulas and the proofs. Therefore we would like to present here systematic and concrete techniques for constructing the primitive characteristic integrals together with new and self-contained proofs.

Furthermore, many works $[14,16,21]$ are concerned with using the characteristic integrals to obtain explicit solutions to the original Toda field theories (1.3) by the method of Darboux. Recently, Anderson, Fels and Vassiliou [1] developed a very general approach to the study of Darboux integrable systems.

By demonstrating enough characteristic integrals, this paper explicitly shows that the Toda field theories (1.3) are Darboux integrable. In sequels to this work, Anderson and the author will apply the characteristic integrals found in this paper and the structure theory in [1] to realize the Toda field theory (1.3) as the quotient of two standard differential systems [20] depending on $x$ and $y$ separately. We will also apply the method and result of this paper to the setting of differential invariants for parabolic geometry [4] with the standard differential system, generalizing works done by Mari Beffa [17].

This paper is organized as follows. In Section 2, we present our main results. Our main theorem is Theorem 2.1, which is applicable to all simple Lie algebras. It employs the zero curvature representation [15] of the Toda field theories (1.3) under a Drinfeld-Sokolov gauge [6]. When the Lie algebra $\mathfrak{g}$ has a non-branching representation (see (2.9)), which is the case for Lie algebras of types $A, B, C$ and $\mathfrak{g}_{2}$, we present a more concrete formula in Theorem 2.2, which is easier to use. We stress that in both cases, we provide complete and novel mathematical proofs.

In Sections 3, we demonstrate the more direct method in Theorem 2.2 in all the applicable cases. In Section 4, we illustrate the general method in Theorem 2.1 by the example of the characteristic 
integral related to the Pffaffian of $D_{4}$. We stress that our constructions are easily implemented using mathematical softwares such as Maple, which this author has done. Maple programs and formulas for the characteristic integrals will be made available at the DifferentialGeometry Software Project website at the Digital Commons of the Utah State Univeristy (http://digitalcommons.usu.edu/dg/). Therefore our results are readily usable for various purposes.

Acknowledgment. The author thanks Ian Anderson for introducing this topic to him. He thanks Anderson and Pawel Nurowski for helps with Maple. The author also thanks László Fehér and Luen-Chau Li for their interests and useful correspondences. He thanks the referee for detailed comments and some references.

\section{Main results}

Let us first introduce some terminology. Let $\mathfrak{h} \subset \mathfrak{g}$ be a Cartan subalgebra, and we denote the corresponding set of roots of $\mathfrak{g}$ by $\Delta$, the sets of positive/negative roots by $\Delta_{ \pm}$, and the set of positive simple roots by $\pi=\left\{\alpha_{i}\right\}_{i=1}^{n}$. Let $\mathfrak{g}=\mathfrak{h} \oplus \bigoplus_{\alpha \in \Delta} \mathfrak{g}_{\alpha}$ be the root space decomposition.

For a root $\alpha$, define its height by $\operatorname{ht}(\alpha)=\sum_{i=1}^{n} c_{i}$ if $\alpha=\sum_{i=1}^{n} c_{i} \alpha_{i}$. Also define the principal height gradation

$$
\mathfrak{g}=\bigoplus_{k=-p}^{p} \mathfrak{g}_{k}, \quad \mathfrak{g}_{k}=\bigoplus_{\mathrm{ht}(\alpha)=k} \mathfrak{g}_{\alpha}, \mathfrak{g}_{0}=\mathfrak{h}
$$

where $p$ is the maximal height of the roots. We also denote by $\mathfrak{n}=\bigoplus_{\alpha \in \Delta_{+}} \mathfrak{g}_{\alpha}=\bigoplus_{k>0} \mathfrak{g}_{k}$ the maximal nilpotent subalgebra, and by $N$ the corresponding unipotent group.

For $\alpha \in \Delta_{+}$, let $e_{\alpha}$ and $e_{-\alpha}$ be root vectors in the root spaces $\mathfrak{g}_{\alpha}$ and $\mathfrak{g}_{-\alpha}$ such that for $H_{\alpha}=$ $\left[e_{\alpha}, e_{-\alpha}\right] \in \mathfrak{h}$, we have $\alpha\left(H_{\alpha}\right)=2$. Then the Cartan matrix $A=\left(a_{i j}\right)_{i, j=1}^{n}$ of $\mathfrak{g}$ is defined by $a_{i j}=$ $\alpha_{i}\left(H_{\alpha_{j}}\right)$.

Let us recall the zero curvature representation of (1.3) following [15]. Let

$$
\mathbf{u}=\sum_{i=1}^{n} u_{x}^{i} H_{\alpha_{i}}, \quad \varepsilon=\sum_{i=1}^{n} e_{-\alpha_{i}}, \quad Y=\sum_{i=1}^{n} e^{\rho_{i}} e_{\alpha_{i}}
$$

where as in (1.3) $\rho_{i}=\sum_{j=1}^{n} a_{i j} u^{j}$. Then the Toda field theory (1.3) is equivalent to the following zero curvature equation

$$
\left[-\partial_{x}+\varepsilon+\mathbf{u}, \partial_{y}+Y\right]=0
$$

Now let us recall the definition of a Kostant slice $\mathfrak{s} \subset \mathfrak{g}$ [13], which is used in a Drinfeld-Sokolov gauge [6]. Let $\mathfrak{s}$ be a complement of $[\varepsilon, \mathfrak{g}]$ in $\mathfrak{g}$, that is,

$$
\mathfrak{g} \cong \mathfrak{s} \oplus[\varepsilon, \mathfrak{g}]
$$

Then by [13], $\mathfrak{s} \subset \mathfrak{n}$, and $\operatorname{dim}(\mathfrak{s})=n$ is equal to the rank. We call $\mathfrak{s}$ a Kostant slice, and let $\left\{s_{j}\right\}_{j=1}^{n}$ be a homogeneous basis of $\mathfrak{s}$ with respect to the height gradation (2.1). 
By [6], we can bring the first element in (2.3) into its Drinfeld-Sokolov gauge. More precisely, there exists an element $g \in N$ (whose components are differential polynomials of the $u^{i}$ ) such that

$$
g\left(-\partial_{x}+\varepsilon+\mathbf{u}\right) g^{-1}=-\partial_{x}+\varepsilon+\mathbf{I}, \quad \mathbf{I}=\sum_{j=1}^{n} I_{j} s_{j} \in \mathfrak{s},
$$

where the components $I_{j}$ are differential polynomials of the $u^{i}$.

Theorem 2.1. For the solutions $u^{i}$ to (1.3), the differential polynomials $I_{j}$ for $1 \leq j \leq n$ defined in (2.5) through a Drinfeld-Sokolov gauge are primitive characteristic integrals, that is, $\partial_{y} I_{j}=0$ and the $I_{j}$ are the generators for the differential polynomial algebra of characteristic integrals.

Proof. Suppose that for the $g \in N$ in (2.5), we have

$$
g\left(\partial_{y}+Y\right) g^{-1}=\partial_{y}+\tilde{Y} .
$$

Since $g \in N$ and $Y \in \mathfrak{g}_{1}$ by (2.2), we have $\tilde{Y} \in \mathfrak{n}$. Suppose $\tilde{Y}=\sum_{i=1}^{p} Y_{i}$ by the height decomposition (2.1).

By the invariance of the zero-curvature equation (2.3) under the adjoint action by $g$, from (2.5) we get

$$
\left[-\partial_{x}+\varepsilon+\sum_{j=1}^{n} I_{j} s_{j}, \partial_{y}+\sum_{i=1}^{p} Y_{i}\right]=0
$$

We will prove by induction that all the $Y_{i}=0$. Clearly this then implies that $\partial_{y} I_{j}=0$ for $1 \leq j \leq n$.

First recall the basic result of Kostant [12] that $\operatorname{ker}\left(\operatorname{ad}_{\mathcal{\varepsilon}}\right) \cap(\mathfrak{h} \oplus \mathfrak{n})=0$. The term on the left of (2.6) with height zero is $\left[\varepsilon, Y_{1}\right]=0$, which then implies that $Y_{1}=0$.

Now assume $i \geq 2$ and that $Y_{j}=0$ for $j \leq i-1$. Then since the $s_{j} \in \mathfrak{n}$, the term on the left of (2.6) with height $i-1$ is

$$
\left[\varepsilon, Y_{i}\right]-\sum_{\mathrm{ht}\left(s_{j}\right)=i-1}\left(\partial_{y} I_{j}\right) s_{j}=0 .
$$

By the decomposition (2.4), we get $\left[\varepsilon, Y_{i}\right]=0$ which then implies that $Y_{i}=0$.

These $I_{j}$ in (2.5) are the generators for the characteristic integrals since without the differentiation operator $-\partial_{x}$, we obtain the generators for the algebraic invariant functions of $\varepsilon+\mathbf{u}$ by [13]. See also [3, Section V].

Remark 2.1. The calculation of $g \in N$ and the $I_{j}$ in (2.5) can be done inductively using the decomposition (2.4) as in [6,7]. For the reader's convenience, we provide some details. Then formula (2.5) is

$$
\partial_{x} g \cdot g^{-1}+g(\varepsilon+\mathbf{u}) g^{-1}=\varepsilon+\mathbf{I} .
$$

Consider

$$
g=e^{a_{1}} \cdots e^{a_{m}}, \quad a_{i} \in \mathfrak{g}_{i}, i=1, \ldots, m .
$$

Inductively for $i \geq 1$, assume that the $a_{j}$ for $1 \leq j \leq i-1$ have been determined. Consider $g_{i-1}=$ $e^{a_{1}} \cdots e^{a_{i-1}}$. Then the component of $\partial_{x} g_{i-1} \cdot g_{i-1}^{-1}+g_{i-1}(\varepsilon+\mathbf{u}) g_{i-1}^{-1}$ with grade $i-1$ can be uniquely written as $\left[\varepsilon, a_{i}\right]+J_{i-1}$ with $J_{i-1} \in \mathfrak{s} \cap \mathfrak{g}_{i-1}$ by (2.4). Then using exactly this $a_{i}$ in (2.7) will do 
the job of the Drinfeld-Sokolov gauging at grade $i-1$. The algorithm is easily implemented on a mathematical software such as Maple, as long as we know the structure equations of the Lie algebra. This author has worked out the characteristic integrals of the $E_{6}$ Toda field theory by this method. In Section 4, we will see the example of $D_{4}$.

Furthermore very often there are more direct formulas for the characteristic integrals as we explain now.

Take an irreducible representation $\phi: \mathfrak{g} \rightarrow$ End $V$. Let the $\beta_{k} \in \mathfrak{h}^{*}$ for $1 \leq k \leq m$ be the weights of $\phi$, and

$$
V=\bigoplus_{k=1}^{m} V_{\beta_{k}}
$$

the weight space decomposition. We assume that $\operatorname{dim} V_{\beta_{k}}=1$ and also that the representation $\phi$ does not branch. That is, for each weight $\beta_{k}$ there is one unique negative simple root $-\alpha_{i_{k}}$ such that $\beta_{k}-\alpha_{i_{k}}$ is another weight of $\phi$. Order our weights such that $\beta_{k+1}=\beta_{k}-\alpha_{i_{k}}$ for $1 \leq k \leq m-1$ and we draw the following weight diagram

$$
\beta_{m} \stackrel{-\alpha_{i_{m-1}}}{\longleftarrow} \cdots \stackrel{-\alpha_{i_{2}}}{\longleftarrow} \beta_{2} \stackrel{-\alpha_{i_{1}}}{\longleftarrow} \beta_{1} .
$$

Such non-branching representations occur for the first fundamental representations of the Lie algebras $A_{n}, B_{n}, C_{n}$ and $\mathfrak{g}_{2}$.

Theorem 2.2. If a non-branching representation $\phi$ as above exists with the weight diagram (2.9), then we have

$$
\left[\left(\partial_{x}-\beta_{1}(\mathbf{u})\right)\left(\partial_{x}-\beta_{2}(\mathbf{u})\right) \cdots\left(\partial_{x}-\beta_{m}(\mathbf{u})\right), \partial_{y}\right]=0,
$$

for a $\mathbf{u}$ (2.2) satisfying (2.3) or equivalently the Toda filed theory (1.3). The product is in the sense of composition for operators on functions of $x$ and $y$, and we strictly follow the order. If by the Leibniz rule, we expand

$$
\left(\partial_{x}-\beta_{1}(\mathbf{u})\right)\left(\partial_{x}-\beta_{2}(\mathbf{u})\right) \cdots\left(\partial_{x}-\beta_{m}(\mathbf{u})\right)=\partial_{x}^{m}+\sum_{j=1}^{m} I_{j} \partial_{x}^{m-j},
$$

then (2.10) implies that

$$
\partial_{y} I_{j}=0, \quad 1 \leq j \leq m .
$$

Furthermore, if the $d_{j}$ for $1 \leq j \leq n$ are the degrees of the simple Lie algebra $\mathfrak{g}$, then the $I_{d_{j}}$ for $1 \leq j \leq n$ are the generators of the differential polynomial algebra of characteristic integrals.

Proof. In the weight decomposition (2.8), we let $v_{\beta_{1}} \in V_{\beta_{1}}$ be a weight vector for the highest weight, and inductively we define the other weight vectors by $v_{\beta_{k}}=\phi\left(e_{-\alpha_{i_{k-1}}}\right) v_{\beta_{k-1}}$ for $2 \leq k \leq m$ by (2.9). Therefore by (2.2)

$$
\phi(\varepsilon) v_{\beta_{k-1}}=v_{\beta_{k}}, \quad 2 \leq k \leq m .
$$

The zero curvature equation (2.3) is the compatibility condition for the following system of equations. Let $\psi(x, y)=\sum_{k=1}^{m} \psi_{k}(x, y) v_{\beta_{k}}$ be a function of $x$ and $y$ with values in $V$. Then (2.3) 
implies that the following system of equations has solutions

$$
\left\{\begin{array}{l}
\left(-\partial_{x}+\phi(\mathbf{u}+\varepsilon)\right) \psi=0 \\
\left(\partial_{y}+\phi(Y)\right) \psi=0 .
\end{array}\right.
$$

Then by (2.13) and $\mathbf{u} \in \mathfrak{h}$ (2.2), the first equation, at the weight vector $v_{\beta_{k}}$, means that

$$
\left(\partial_{x}-\beta_{k}(\mathbf{u})\right) \psi_{k}=\psi_{k-1}, \quad 2 \leq k \leq m .
$$

When $k=1$, we actually have

$$
\left(\partial_{x}-\beta_{1}(\mathbf{u})\right) \psi_{1}=0
$$

since $\beta_{1}$ is the highest weight. Therefore combining them, we have

$$
\left(\partial_{x}-\beta_{1}(\mathbf{u})\right)\left(\partial_{x}-\beta_{2}(\mathbf{u})\right) \cdots\left(\partial_{x}-\beta_{m}(\mathbf{u})\right) \psi_{m}=0 .
$$

On the other hand, the second equation in (2.14), at the lowest weight vector $v_{\beta_{m}}$, quickly gives that

$$
\partial_{y} \psi_{m}=0,
$$

since $Y \in \mathfrak{n}$ (2.2). Therefore the above two equations have a solution $\psi_{m}=\psi_{m}(x, y)$, which is general. The implied compatibility condition is exactly (2.10), which quickly gives (2.12).

In the expansion (2.11), if we replace the differential operator $\partial_{x}$ by an algebraic indeterminant $\lambda$ then the corresponding $I_{d_{j}}$, corresponding to the degrees $d_{j}$ of $\mathfrak{g}$ for $1 \leq j \leq n$, are well-known to be the generators for the algebraic invariant functions of $\mathbf{u}$. Therefore the current $I_{d_{j}}$ in (2.11) with the differential operator are independent and are the generators for the characteristic integrals.

\section{Examples for Theorem 2.2}

The first fundamental representations of the Lie algebras $A_{n}, B_{n}, C_{n}$ and $\mathfrak{g}_{2}$ are non-branching representations (2.9). Therefore Theorem 2.2 applies to these situations and we obtain their primitive characteristic integrals as corollaries.

Keeping our spirit of being as explicit as possible, we present the formulas in these cases. In this section we follow [9] for notation and choices of root vectors. The Cartan subalgebras $\mathfrak{h}$ always consist of diagonal matrices. We let $L_{i} \in \mathfrak{h}^{*}$ denote the linear function of taking the $i$ th element on the diagonal. We also let $E_{i j}$ denote the matrix with a 1 at the $(i, j)$-position and zero everywhere else. For simplicity, we write $\partial$ for $\partial_{x}$.

Example 3.1 $\left(A_{n}\right)$. The simple roots for $A_{n}$ are $\alpha_{i}=L_{i}-L_{i+1}$ and the $H_{\alpha_{i}}=E_{i, i}-E_{i+1, i+1}, 1 \leq i \leq n$. The Cartan matrix is

$$
\left(\begin{array}{ccccc}
2 & -1 & & & \\
-1 & 2 & -1 & & \\
& \ddots & \ddots & \ddots & \\
& & -1 & 2 & -1 \\
& & & -1 & 2
\end{array}\right)
$$

The degrees of $A_{n}$ (that is, the degrees of primitive adjoint-invariant functions) are $2,3, \cdots, n+1$. The weight diagram for the first fundamental representation is

$$
L_{n+1} \stackrel{-\alpha_{n}}{\longleftarrow} \cdots \stackrel{-\alpha_{2}}{\longleftarrow} L_{2} \stackrel{-\alpha_{1}}{\longleftarrow} L_{1} .
$$


The $\mathbf{u}$ in (2.2) is

$$
\mathbf{u}=\operatorname{Diag}\left(u_{x}^{1}, u_{x}^{2}-u_{x}^{1}, \cdots, u_{x}^{n}-u_{x}^{n-1},-u_{x}^{n}\right)
$$

By Theorem 2.2, consider the expansion

$$
\begin{aligned}
& \left(\partial-u_{x}^{1}\right)\left(\partial+u_{x}^{1}-u_{x}^{2}\right) \cdots\left(\partial+u_{x}^{n-1}-u_{x}^{n}\right)\left(\partial+u_{x}^{n}\right) \\
= & \partial^{n+1}+\sum_{j=1}^{n} I_{j} \partial^{n-j} .
\end{aligned}
$$

Then the $I_{j}$ for $1 \leq j \leq n$ are primitive characteristic integrals of the $A_{n}$ Toda field theory (1.3).

Example 3.2 $\left(C_{n}, n \geq 2\right)$. The simple roots for $C_{n}$ are $\alpha_{i}=L_{i}-L_{i+1}$ for $1 \leq i \leq n-1$ and $\alpha_{n}=2 L_{n}$. Also $H_{\alpha_{i}}=E_{i, i}-E_{i+1, i+1}-E_{n+i, n+i}+E_{n+i+1, n+i+1}$ for $1 \leq i \leq n-1$ and $H_{\alpha_{n}}=E_{n, n}-E_{2 n, 2 n}$. The Cartan matrix is

$$
\left(\begin{array}{ccccc}
2 & -1 & & & \\
-1 & 2 & -1 & & \\
& \ddots & \ddots & \ddots & \\
& & -1 & 2 & -1 \\
& & & -2 & 2
\end{array}\right)
$$

The degrees of $C_{n}$ are $2,4, \cdots, 2 n$. The weight diagram for the first fundamental representation is

$$
-L_{1} \stackrel{-\alpha_{1}}{\longleftarrow} \cdots \stackrel{-\alpha_{n-1}}{\longleftarrow}-L_{n} \stackrel{-\alpha_{n}}{\longleftarrow} L_{n} \stackrel{-\alpha_{n-1}}{\longleftarrow} \cdots \stackrel{-\alpha_{2}}{\longleftarrow} L_{2} \stackrel{-\alpha_{1}}{\longleftarrow} L_{1} .
$$

The $\mathbf{u}$ in (2.2) is

$$
\mathbf{u}=\operatorname{Diag}\left(u_{x}^{1}, u_{x}^{2}-u_{x}^{1}, \cdots, u_{x}^{n}-u_{x}^{n-1},-u_{x}^{1},-u_{x}^{2}+u_{x}^{1}, \cdots,-u_{x}^{n}+u_{x}^{n-1}\right) .
$$

By Theorem 2.2, consider the expansion

$$
\begin{aligned}
& \left(\partial-u_{x}^{1}\right)\left(\partial+u_{x}^{1}-u_{x}^{2}\right) \cdots\left(\partial+u_{x}^{n-1}-u_{x}^{n}\right) \\
& \left(\partial+u_{x}^{n}-u_{x}^{n-1}\right) \cdots\left(\partial+u_{x}^{2}-u_{x}^{1}\right)\left(\partial+u_{x}^{1}\right) \\
= & \partial^{2 n}+\sum_{j=1}^{n} I_{j} \partial^{2 n-2 j}+\sum_{j=1}^{n-1} J_{j} \partial^{2 n-2 j-1} .
\end{aligned}
$$

Then the $I_{j}$ for $1 \leq j \leq n$ are primitive characteristic integrals of the $C_{n}$ Toda field theory, and the $J_{j}$ are some differential polynomials in them.

Example 3.3 $\left(B_{n}, n \geq 2\right)$. The simple roots for $B_{n}$ are $\alpha_{i}=L_{i}-L_{i+1}$ for $1 \leq i \leq n-1$ and $\alpha_{n}=L_{n}$. Also $H_{\alpha_{i}}=E_{i, i}-E_{i+1, i+1}-E_{n+i, n+i}+E_{n+i+1, n+i+1}$ for $1 \leq i \leq n-1$ and $H_{\alpha_{n}}=2 E_{n, n}-2 E_{2 n, 2 n}$. The 
Cartan matrix is

$$
\left(\begin{array}{cccccc}
2 & -1 & & & \\
-1 & 2 & -1 & & \\
& \ddots & \ddots & \ddots & \\
& & -1 & 2 & -2 \\
& & & -1 & 2
\end{array}\right)
$$

The degrees of $B_{n}$ are $2,4, \cdots, 2 n$. The weight diagram for the first fundamental representation is

$$
-L_{1} \stackrel{-\alpha_{1}}{\longleftarrow} \cdots \stackrel{-\alpha_{n-1}}{\longleftarrow}-L_{n} \stackrel{-\alpha_{n}}{\longleftarrow} 0 \stackrel{-\alpha_{n}}{\longleftarrow} L_{n} \stackrel{-\alpha_{n-1}}{\longleftarrow} \cdots \stackrel{-\alpha_{2}}{\longleftarrow} L_{2} \stackrel{-\alpha_{1}}{\longleftarrow} L_{1} .
$$

The $\mathbf{u}$ in (2.2) is

$$
\mathbf{u}=\operatorname{Diag}\left(u_{x}^{1}, u_{x}^{2}-u_{x}^{1}, \cdots, 2 u_{x}^{n}-u_{x}^{n-1},-u_{x}^{1},-u_{x}^{2}+u_{x}^{1}, \cdots,-2 u_{x}^{n}+u_{x}^{n-1}, 0\right) .
$$

By Theorem 2.2, consider the expansion

$$
\begin{aligned}
& \left(\partial-u_{x}^{1}\right)\left(\partial+u_{x}^{1}-u_{x}^{2}\right) \cdots\left(\partial+u_{x}^{n-1}-2 u_{x}^{n}\right) \\
& \partial\left(\partial+2 u_{x}^{n}-u_{x}^{n-1}\right) \cdots\left(\partial+u_{x}^{2}-u_{x}^{1}\right)\left(\partial+u_{x}^{1}\right) \\
= & \partial^{2 n+1}+\sum_{j=1}^{n} I_{j} \partial^{2 n-2 j+1}+\sum_{j=1}^{n} J_{j} \partial^{2 n-2 j} .
\end{aligned}
$$

Then the $I_{j}$ for $1 \leq j \leq n$ are primitive characteristic integrals of the $B_{n}$ Toda field theory, and the $J_{j}$ are some differential polynomials in them.

Example $3.4\left(\mathfrak{g}_{2}\right)$. The Cartan matrix is

$$
\left(\begin{array}{cc}
2 & -1 \\
-3 & 2
\end{array}\right)
$$

For simplicity we call the unknown functions $u^{1}, u^{2}$ by $u$ and $v$. The Toda system is

$$
\left\{\begin{array}{l}
u_{x y}=-e^{2 u-v} \\
v_{x y}=-e^{-3 u+2 v}
\end{array}\right.
$$

Although we can work abstractly with just the above Cartan matrix, we choose to use the embedding of $\mathfrak{g}_{2} \subset \mathfrak{s o}_{7}=B_{3}$ such that the two roots are

$$
\alpha_{1}=L_{1}-L_{2}, \quad \alpha_{2}:=L_{2}-L_{3},
$$

and

$$
\begin{aligned}
& H_{\alpha_{1}}=\operatorname{Diag}(1,-1,2,-1,1,-2,0) \\
& H_{\alpha_{2}}=\operatorname{Diag}(0,1,-1,0,-1,1,0) .
\end{aligned}
$$

The $\mathbf{u}$ in (2.2) is

$$
\mathbf{u}=\operatorname{Diag}\left(u_{x},-u_{x}+v_{x}, 2 u_{x}-v_{x},-u_{x}, u_{x}-v_{x},-2 u_{x}+v_{x}, 0\right) .
$$

The first fundamental representation of $\mathfrak{g}_{2}$ is the restriction of that of $B_{3}$. Therefore we follow the weight diagram (3.3). The degrees of $\mathfrak{g}_{2}$ are 2 and 6 . 
By Theorem 2.2, consider the expansion

$$
\begin{gathered}
\left(\partial-u_{x}\right)\left(\partial+u_{x}-v_{x}\right)\left(\partial-2 u_{x}+v_{x}\right) \\
\partial\left(\partial+2 u_{x}-v_{x}\right)\left(\partial-u_{x}+v_{x}\right)\left(\partial+u_{x}\right) \\
=\partial^{7}+I_{1} \partial^{5}+I_{2} \partial+\sum_{j=1}^{3} J_{j} \partial^{5-j}+J_{4} .
\end{gathered}
$$

Then the $I_{1}$ and $I_{2}$ are primitive characteristic integrals of the $\mathfrak{g}_{2}$ Toda field theory, and the $J_{j}$ are some differential polynomials in them.

\section{An example of Theorem 2.1}

It can be said that Theorem 2.2 is a quick way to compute a Drinfeld-Sokolov gauge (2.5) when there is a non-branching representation, and this is the viewpoint in [3]. When such a simple representation does not exist, a Drinfeld-Sokolov gauge can still be computed through an inductive procedure by Remark 2.1 so as to yield the characteristic integrals by Theorem 2.1. We stress that the Drinfeld-Sokolov gauging can be achieved intrinsically using just the adjoint representation, as this author has done for the $E_{6}$ Toda field theory.

In this section, we show as an example how to construct the characteristic integrals for $D_{4}$, where an explicit matrix presentation of the Lie algebra is easy to write down which makes the calculation more explicit. That we indeed obtain the characteristic integrals in this example is a corollary of Theorem 2.1.

First recall that the Cartan matrix of $D_{n}$ is

$$
\left(\begin{array}{cccccc}
2 & -1 & & & & \\
-1 & 2 & -1 & & & \\
& \ddots & \ddots & \ddots & & \\
& & -1 & 2 & -1 & -1 \\
& & & -1 & 2 & \\
& & & -1 & & 2
\end{array}\right)
$$

and its first fundamental representation branches with the following weight diagram

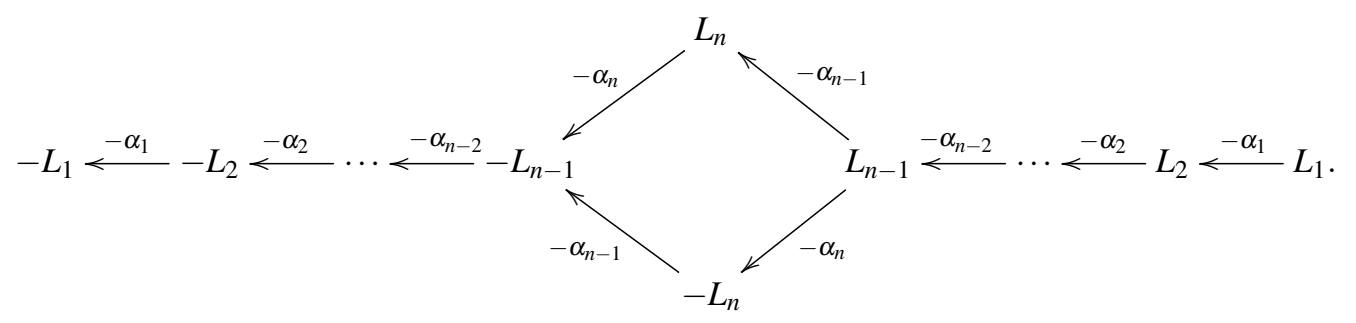


Therefore Theorem 2.2 can not be applied to the Lie algebras $D_{n}$, but Theorem 2.1 can as we will do below to the example of $D_{4}$.

Example 4.1 $\left(D_{4}\right)$. For simplicity, we write $u, v, w, z$ for the unknown functions $u^{1}, \cdots, u^{4}$. The Toda field theory (1.3) for $D_{4}$ is

$$
\left\{\begin{array}{l}
u_{x y}=-e^{2 u-v} \\
v_{x y}=-e^{-u+2 v-w-z} \\
w_{x y}=-e^{-v+2 w} \\
z_{x y}=-e^{-v+2 z} .
\end{array}\right.
$$

The degrees of $D_{4}$ are 2,4,4,6, with the Pfaffian, the square root of the determinant, being an extra adjoint-invariant function of degree 4.

In this example, we use the convention that the non-degenerate symmetric matrix preserved by the orthogonal matrices in $S O(8)$ is the anti-diagonal matrix $\left(\delta_{i, 9-j}\right)_{i, j=1}^{8}$. Then the matrices in $D_{4}=\mathfrak{s o}_{8}$ are skew-symmetric with respect to the anti-diagonal. For simplicity, we use the notation $u_{1}=u_{x}, u_{2}=u_{x x}, u_{3}=u_{x x x}$ and so on. The $\varepsilon+\mathbf{u}$ from (2.2) is

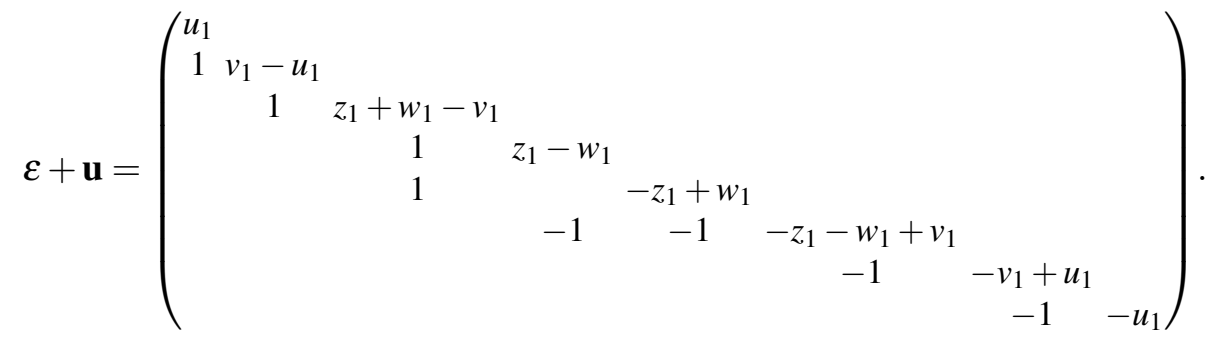

We choose our slice in (2.5) to be

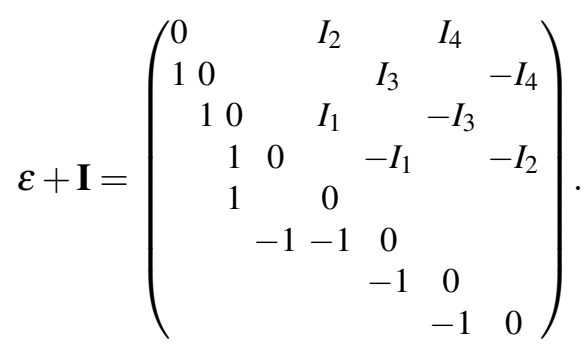

A unipotent orthogonal matrix $B \in N_{S O(8)}$ is an upper-triangular matrix with 1's on the diagonal and element $b_{i, j}$ at the position $(i, j)$ for $i<j$ satisfying some extra conditions for the orthogonality. In particular we have that $b_{4,5}=0$ and $b_{3,4}+b_{5,6}=0$. (Actually one can write out the $B$ using its affine coordinates and solving the orthogonality conditions.) Solving (2.5) by Maple, we get the following expression for the primitive characteristic integrals of the $D_{4}$ Toda field theory.

$$
\begin{gathered}
I_{1}=-\left(u_{2}+v_{2}+z_{2}+w_{2}-u_{1}^{2}-v_{1}^{2}-z_{1}^{2}-w_{1}^{2}+u_{1} v_{1}+z_{1} v_{1}+w_{1} v_{1}\right) \\
I_{2}=u_{4}+v_{4}+2 w_{4}+z_{2} v_{1}^{2}+3 w_{2} v_{2}-w_{2} v_{1}^{2}+w_{1}^{2} v_{2}+v_{2} z_{2} \\
-v_{2} z_{1}^{2}+2 v_{2} u_{2}-z_{2} u_{2}-w_{2} u_{1}^{2}+z_{2} u_{1}^{2}+v_{3} u_{1} \\
-2 u_{3} u_{1}+2 v_{3} w_{1}-w_{1}^{2} u_{2}+w_{2} u_{2}-4 w_{2}^{2}-2 v_{2}^{2} \\
-2 u_{2}^{2}+z_{1}^{2} u_{2}+w_{2} v_{1} u_{1}-z_{1} v_{1} u_{2}+w_{1} v_{1} u_{2} \\
\text { Co-published by Atlantis Press and Taylor \& Francis } \\
\text { Copyright: the authors }
\end{gathered}
$$




$$
\begin{aligned}
& -z_{2} v_{1} u_{1}-2 v_{1} w_{1} v_{2}+2 w_{2} v_{1} w_{1}+2 z_{1} v_{1} v_{2}-2 v_{1} z_{1} z_{2} \\
& +u_{3} v_{1}-2 v_{1} v_{3}+v_{1} z_{3}-4 w_{1} w_{3}+v_{1} w_{3}-z_{1}^{2} u_{1}^{2}+w_{1}^{2} u_{1}^{2} \\
& -w_{1}^{2} v_{1} u_{1}-z_{1} v_{1}^{2} u_{1}-w_{1} v_{1} u_{1}^{2}+z_{1}^{2} v_{1} u_{1}+z_{1} v_{1} u_{1}^{2}+w_{1} v_{1}^{2} u_{1} \\
I_{3} & =2 u_{4}+v_{4}+w_{4}+w_{1}{ }^{2} u_{1}^{2}-2 v_{1} v_{3}+v_{1} z_{3}-2 w_{1} w_{3}+v_{1} w_{3} \\
& +u_{3} v_{1}+3 v_{2} u_{2}-w_{2} u_{1}^{2}+w_{2} u_{2}+2 v_{3} u_{1}-4 u_{3} u_{1}+v_{3} w_{1} \\
& -w_{1}^{2} u_{2}+v_{2} z_{2}-v_{2} z_{1}^{2}+z_{2} v_{1}^{2}+2 w_{2} v_{2}-2 v_{2}^{2}-4 u_{2}^{2} \\
& -2 w_{2}^{2}-w_{1}{ }^{2} v_{1} u_{1}+w_{1} v_{1}^{2} u_{1}+2 z_{1} v_{1} v_{2}-2 v_{1} z_{1} z_{2}+w_{1} v_{1} u_{2} \\
& +w_{2} v_{1} u_{1}-w_{1} v_{1} u_{1}^{2}-v_{1}^{2} u_{2}+z_{1}^{2} w_{2}-z_{1}^{2} w_{1}^{2}+v_{2} u_{1}^{2}-z_{2} w_{2} \\
& +z_{2} w_{1}^{2}+z_{1}^{2} v_{1} w_{1}-v_{1}^{2} z_{1} w_{1}+w_{1}^{2} v_{1} z_{1}-z_{2} v_{1} w_{1}-w_{2} v_{1} z_{1} \\
& +2 v_{1} u_{2} u_{1}-2 v_{2} v_{1} u_{1} \\
I_{4} & =-u_{6}-\frac{1}{2} v_{6}-w_{6}+\text { a lot of other terms which we omit }
\end{aligned}
$$

Actually the usual Pfaffian of $\varepsilon+\mathbf{u}$ in (4.2), which is the product of the first 4 diagonal entries, is contained in the characteristic integral $I_{2}$ as the last terms involving only partial derivatives of the first order.

Remark 4.1. In [3], there is a procedure to apply an integration step in using the first fundamental representation (4.1) of $D_{n}$ to get an analogous formula to Theorem 2.2. That integration step will cause us to lose one characteristic integral, which in the case of $D_{4}$ is exactly the above $I_{2}$ corresponding to the Pfaffian.

\section{References}

[1] Ian M. Anderson, Mark E. Fels and Peter J. Vassiliou, Superposition formulas for exterior differential systems, Adv. Math. 221 (2009) 1910-1963.

[2] Olivier Babelon,Denis Bernard and Michel Talon, Introduction to classical integrable systems, Cambridge Monographs on Mathematical Physics (Cambridge University Press, Cambridge, 2003).

[3] J. Balog, L. Fehér, L. O'Raifeartaigh, P. Forgács and A. Wipf, Toda theory and $\mathcal{W}$-algebra from a gauged WZNW point of view, Ann. Physics 203 (1990) 76-136.

[4] Andreas Čap and Jan Slovák, Parabolic geometries. I, Mathematical Surveys and Monographs 154 (American Mathematical Society, Providence, RI, 2009), background and general theory.

[5] D.K. Demskoi, Integrals of open two-dimensional lattices, Theoret. and Math. Phys. 163 (2010) 466471.

[6] V.G. Drinfel'd and V.V. Sokolov, Lie algebras and equations of Korteweg-de Vries type, Current Problems in Mathematics 24 (Itogi Nauki i Tekhniki,Akad. Nauk SSSR Vsesoyuz. Inst. Nauchn. i Tekhn. Inform., Moscow, 1984) 81-180, Russian.

[7] L. Fehér, L. O'Raifeartaigh, P. Ruelle, I. Tsutsui and A. Wipf, Generalized Toda theories and $\mathcal{W}$ algebras associated with integral gradings, Ann. Physics 213 (1992) 1-20.

[8] Boris Feigin and Edward Frenkel, Integrals of motion and quantum groups, Integrable systems and quantum groups (Montecatini Terme, 1993), Lecture Notes in Math. 1620 (Springer, Berlin, 1996) 349-418.

[9] William Fulton and Joe Harris, Representation theory, Graduate Texts in Mathematics 129 (SpringerVerlag, New York, 1991), a first course; Readings in Mathematics.

[10] A.M. Gur'eva and A.V. Zhiber, Laplace invariants of two-dimensionalized open Toda chains, Teoret. Mat. Fiz. 138 (2004) 401-421, Russian, with Russian summary; English translation: Theoret. and Math. Phys. 138 (2004) 338-355. 
[11] A.M. Gur'eva and A.V. Zhiber, Laplace invariants of Toda lattices with exceptional Cartan matrices, http: //arxiv.org/abs/nlin/0512001 (2005).

[12] Bertram Kostant, The principal three-dimensional subgroup and the Betti numbers of a complex simple Lie group, Amer. J. Math. 81 (1959) 973-1032.

[13] Bertram Kostant, Lie group representations on polynomial rings, Amer. J. Math. 85 (1963) $327-404$.

[14] Erling G.B. Hohler and Kåre Olaussen, Using conservation laws to solve Toda field theories, Internat. J. Modern Phys. A 11 (1996) 1831-1853.

[15] A.N. Leznov and M.V. Saveliev, Representation of zero curvature for the system of nonlinear partial differential equations $x_{\alpha, z \bar{z}}=\exp (k x)_{\alpha}$ and its integrability, Lett. Math. Phys. 3 (1979) 489-494.

[16] A.N. Leznov and M.V. Saveliev, Group-theoretical methods for integration of nonlinear dynamical systems, Progress in Physics 15 (Birkhäuser Verlag, Basel, 1992), translated and revised from Russian by D.A. Leuites.

[17] G. Mari Beffa, Geometric Hamiltonian structures on flat semisimple homogeneous manifolds, Asian J. Math. 12 (2008) 1-33.

[18] A.B. Shabat, Higher symmetries of two-dimensional lattices, Phys. Lett. A 200 (1995) 121-133.

[19] A.B. Shabat and R. L. Yamilov, Exponential systems of type II and Cartan matrices, Preprint No. 1 Bashkir Branch USSR Acad. Sci. Ufa (1981).

[20] Keizo Yamaguchi, Differential systems associated with simple graded Lie algebras, Progress in differential geometry, Adv. Stud. Pure Math. 22 (Math. Soc. Japan, Tokyo, 1993) 413-494.

[21] A.V. Zhiber and V.V. Sokolov, Exactly integrable hyperbolic equations of Liouville type, Uspekhi Mat. Nauk 56 (2001) 63-106, Russian, with Russian summary; English translation: Russian Math. Surveys 56 (2001) 61-101. 